\title{
Herramientas web 2.0 como apoyo para la formación en educación superior
}

\author{
William Enrique Lara Castro*, Mayerlin del Carmen Cogollo López ${ }^{* *}$ y Abel Nonato Royo Galeano**
}

\section{Resumen}

En el mundo actual, en el que la tecnología ocupa un lugar importante, el principal reto de la docencia radica en la incorporación de las herramientas web 2.0 como medios de apoyo para la educación. Por lo tanto, los cambios en los modelos educativos en los últimos años han motivado a que los docentes adopten el uso de las herramientas web 2.0 en su quehacer diario para producir cambios significativos en el aprendizaje de los alumnos. Este artículo, en el que se buscó determinar si los factores de edad, nivel académico y conocimiento son determinantes en el uso de las herramientas web 2.0, se construyó a partir de un enfoque metodológico de naturaleza cuantitativa de nivel no experimental, que tiene a las herramientas web 2.0 como variable y la encuesta como instrumento de recolección de datos. Como resultados, se encontró que no muchos docentes utilizan estas herramientas y que, cuando lo hacen, prefieren utilizar redes sociales, blogs, microblogs y, en menor medida, wikis, porque permiten el desarrollo de más estrategias pedagógicas y les brindan un espacio de interacción, conexión, reflexión y colaboración.

\section{Abstract}

In today's world, in which technology occupies an important place, the main challenge for teaching lies in the incorporation of web 2.0 tools as a means of support for education. Therefore, changes in educational models in recent years have motivated teachers to adopt the use of web 2.0 tools in their daily work to produce significant changes in student learning. This paper, in which it was sought to determine whether the factors of age, academic level and knowledge are decisive in the use of web 2.0 tools, was built from a methodological approach of a quantitative nature at a non-experimental level, which has the web 2.0 tools as a variable and the survey as a data collection instrument. As a result, it was found that not many teachers use these tools and that, when they do, they prefer to use social networks, blogs, microblogs and, to a lesser extent, wikis, because they allow the development of more pedagogical strategies and provide them with a space for interaction, connection, reflection and collaboration.
Cómo citar este artículo (APA): Lara, W., Cogollo, M., Royo, A. (2019). Herramientas web 2.0 como apoyo para la formación en educación superior. Hashtag, 14, 67-84.

> Palabras clave: constructivismo social, educación superior, enseñanza superior, tecnología de la información, web 2.0

> Keywords: higher education, information technology, postsecondary education, social constructivism, web 2.0

\footnotetext{
* Administrador de Empresas y magíster en Ciencias de la Educación Virtual. Docente de tiempo completo en la Corporación Unificada Nacional de Educación Superior (CUN) - Regional Córdoba. Contacto: william_lara@cun.edu.co

** Administradora Financiera y especialista en Gerencia Empresarial. Administradora Financiera. Docente de medio tiempo en la Corporación Unificada Nacional de Educación Superior (CUN) - Regional Córdoba. Contacto: mayerlin_cogollo@cun.edu.co

*** Administrador de Empresas y magíster en Administración de Empresas. Docente de tiempo completo en la Corporación Unificada Nacional de Educación Superior (CUN) - Regional Córdoba. Contacto: abel_nonato@cun.edu.co
} 


\section{Introducción}

$E^{n}$ los últimos años, el avance en la tecnología ha conllevado el progreso de distintas áreas de la sociedad; por ejemplo, en el campo educativo, la irrupción de las Tecnologías de la Información y la Comunicación (TIC) en las aulas de clase permite poner en práctica distintos métodos de enseñanza-aprendizaje. Cada día, las TIC (y particularmente las herramientas web 2.0) se presentan como una necesidad para un mundo en el que los rápidos cambios, el aumento de los saberes y las demandas de una educación de un alto nivel, constantemente actualizada, se transformen en una exigencia permanente. La relación entre las TIC y la educación tiene dos vertientes: por un lado, los ciudadanos se ven abocados a conocer y aprender sobre las TIC y, por el otro, estas pueden aplicarse al proceso educativo (Rosario, 2006). Dentro de este marco, desde el siglo pasado, la educación superior se ha apostado en un "modelo de enseñanza tradicional basado en las clases magistrales del docente, en la toma de apuntes por parte del alumnado y en la lectura memorizada -y memorización- de una serie de textos bibliográficos por parte de estos" (Area, 2000, p. 128); en otras palabras, simplemente una transmisión pasiva de conocimientos.

Por lo tanto, es válido resaltar la importancia que ejercen las TIC como medios adecuados de comunicación y transmisión de información, ya sea de forma asincrónica o sincrónica, para la interacción entre estudiantes y docentes en la educación (Hernández, G., 2008). Por otra parte, Cabero (2001) afirma que incorporar la tecnología en la educación se ha vuelto casi una necesidad, pero no porque sea una moda o porque todo el mundo hable de ello, sino porque no hacerlo significaría la exclusión de una realidad latente, debido a que esta se encuentra insertada en casi todas las actividades cotidianas del hombre. Esto denota la importancia de la aplicación de las TIC en el proceso educativo y la implicación de sus actores. No obstante, aunque se conozcan los efectos de mejoras que parecen producir el uso de las herramientas web 2.0 en la educación, es fundamental que se investigue qué herramientas web 2.0 utilizan los docentes para desarrollar este avance en la tecnología.

La web 2.0 suministra una gran variedad de herramientas para ser aplicadas en la educación en relación con la sencillez para su aplicación. Conocer el uso de las herramientas web 2.0 por parte de los docentes es de gran importancia, ya que aborda un tema de actualidad y relevancia. Además, la web 2.0 ha ocupado un lugar privilegiado en los sistemas educativos y en la labor del docente durante los últimos años.Toda vez que ya existen investigaciones y aportaciones teóricas que resaltan los beneficios e impactos de las herramientas web 2.0 en la educación superior, el propósito de este estudio se centra en conocer el uso, tipo y aplicación de las herramientas web $2.0^{1}$, como medios didácticos de apoyo para la construcción de conocimiento en el proceso de aprendizaje de los estudiantes de una institución de educación superior de la ciudad de Montería, por parte de los docentes.

En ese sentido, se consideró pertinente indagar sobre los siguientes aspectos vinculados a las herramientas web 2.0: 1) Detectar el grado de conocimiento de las herramientas web 2.0 que tienen los docentes; 2) conocer qué herramientas web 2.0 son utilizadas por los docentes como medio didáctico de apoyo a la formación del estudiante; 3) saber cómo utiliza el docente las herramientas web 2.0 para la construcción de conocimiento, y

1 Se hace referencia a los blogs, wikis, redes sociales, microblogs, herramientas de colaboración, servicios de video, pódcast, marcadores sociales y mapas mentales. 
4) identificar las herramientas web 2.0 más usadas por los docentes.

La revisión de la literatura de los estudios realizados en diferentes latitudes permite evidenciar las experiencias y opiniones de algunos autores acerca de la web 2.0 y los recursos que la conforman, y su uso por docentes y alumnos en el contexto de la educación superior.

En el ámbito europeo sobre la web 2.0 en la educación superior, es de gran importancia un estudio realizado en la Universidad de Huelva España sobre la formación, actitud, uso e impacto de la web 2.0 en educación superior. Boza y Conde ponen de manifiesto que

En relación a [sic] la actitud de los profesores ante la web 2.0, en general nuestros universitarios conciben que la web 2.0 es un instrumento necesario para una enseñanza de calidad, que los docentes más jóvenes están más predispuestos para su uso, que las herramientas de la web 2.0 resultan atractivas y novedosas para los alumnos y, que la web
2.0 hace que el trabajo, tanto para profesores como para alumnos, resulte más fácil. (2015, p. 55)

En Colombia, el artículo "Creación, implementación y validación de un modelo de aprendizaje virtual para la educación superior en tecnologías web 2.0" de Zambrano y Medina (2010), presenta la importancia de un modelo de educación virtual, el cual se fundamenta en el uso de la web 2.0 para la educación superior, ante la imperante necesidad de la producción de conocimiento. Esta producción de conocimiento se describe en la combinación del uso de otras modalidades de educación, en especial con las clases de modalidad presencial. Por su parte, Acosta (2012) desarrolló otro estudio, en la región caribe colombiana, en el que hace referencia al uso de los servicios y herramientas de la web 2.0 por parte de los docentes de planta de la Universidad Tecnológica de Bolívar; allí, el autor explica varios aspectos, por ejemplo, que las aptitudes para el uso y el conocimiento de la web 2.0 están muy dispersas, entre el rango bajo y alto.

\section{Metodología}

Este estudio es de naturaleza cuantitativa, ya que el propósito principal de este enfoque permite recopilar muchos datos numéricos para medir el comportamiento social de los docentes frente al uso de las herramientas web 2.0, y se orienta hacia analizar datos empíricos (medibles u observables) sobre variables (Hernández, Fernández y Baptista, 2010); en este caso, el uso de las herramientas web 2.0 como medio didáctico de apoyo a la formación por parte de los docentes. De otro lado, el alcance es no experimental, exploratorio y descriptivo, porque solo se busca "especificar las propiedades, características y los perfiles de personas [...] que se someta a un análisis" (p. 80). Particularmente, en este estudio solo se pretende recoger información sobre la

situación de los docentes en relación con las herramientas web 2.0 que utilizan y cómo las usan, indicando cómo se relacionan, buscan y recopilan información, considerando el contexto de los participantes en la investigación.

El estudio se lleva a cabo en una Institución de Educación Superior. La exploración se realiza específicamente en los programas de Administración de Empresas, Administración de la Seguridad Social, Contaduría Pública e Ingeniería de Sistemas, que están incorporados en la oferta académica de la institución. Así, se determinó que noventa y tres (93) docentes pertenecían a los programas anteriormente mencionados (en la tabla 1 se muestra cómo están agrupados de 
acuerdo con cada programa). En el estudio se utilizó una muestra de participantes voluntarios, en consecuencia, fue una muestra por conveniencia. En este sentido, luego de analizar y delimitar la población objeto de estudio, el siguiente paso fue la localización específica de las unidades que participaron en la descripción de los eventos fundamentales para el desarrollo del proceso investigativo.

Tabla 1. Distribución de la población de estudio.

\begin{tabular}{cc} 
Escuela académica & $\begin{array}{c}\text { n. }{ }^{\circ} \text { de } \\
\text { docentes }\end{array}$ \\
\hline Administración de Empresas & 28 \\
\hline Administración de la Seguridad Social & 21 \\
\hline Contaduría Pública & 27 \\
\hline Tngeniería de Sistemas & 17 \\
\hline
\end{tabular}

Fuente: elaboración propia.

Para esta investigación, se elaboró un instrumento, en correspondencia con los aspectos centrales planteados en el propósito del estudio, que se formuló con la intención de conocer las herramientas web 2.0 utilizadas por los docentes en las prácticas académicas. Igualmente, fue necesario adoptar la recolección sistemática de la información sobre la población voluntaria; para ello, se utilizó la técnica de la encuesta, la cual "se caracteriza porque la información debe ser obtenida a través de preguntas a otras personas" (López, 2011, p. 192). A través de la encuesta se explora el uso de las herramientas web 2.0 por los docentes, en base a la identificación del perfil para el uso de las mencionadas herramientas.

El diseño de la encuesta para recolección de los datos se realizó con las opciones que ofrece el formulario de Google. Además, esta se aplicó en línea: el enlace se envió vía correo institucional a cada docente. Este modo de realizar la encuesta permitió llegar a toda la población identificada para el estudio. Ahora bien, antes de enviar el enlace se validó el contenido del instrumento. Este fue validado por un especialista en Administración de la Informática Educativa y uno en investigación (también docente de la catedra de opción de grado), quienes dieron sus respectivos juicios de valor sobre las preguntas. Esto permitió proceder a la corrección del instrumento.

Una vez el enlace en el que podían responder la encuesta fue recibido por los docentes, se dio un plazo de cinco (5) días para que la diligenciaran. Después del plazo, se extrajo la información, procesada automáticamente por la herramienta docs.google.com de Google, en una tabla de Excel y, luego, se procedió a interpretarla. En este paso, se valoraron los datos recogidos en relación con el objetivo general del estudio y se demostró que la muestra elegida, el tipo de instrumento empleado para la recolección, el tiempo de aplicación y la conveniencia de la aplicación en línea fueron decisiones acertadas. Las unidades 
de contenido, producto de la depuración del cúmulo de datos logrados mediante el trabajo de campo, sintetizan y describen los hechos, las vivencias, los fenómenos, las situaciones y las conductas de las personas involucradas en el problema planteado. Esta información de carácter puramente descriptivo fue organizada, documentada y analizada de acuerdo con el proceso que exige la investigación cuantitativa.

\section{Resultados}

\section{Información demográfica}

La encuesta fue diligenciada por 50 docentes que, en términos demográficos, se clasificaron de la siguiente manera: La mayoría de los docentes encuestados son del género masculino, con un $68 \%$ de participación (34 docentes), mientras que el género femenino está representado por el $32 \%$ de los docentes (16). En cuanto a la edad, los docentes se encuentran en un rango entre 25 y 64 años, destacando que el mayor número se encuentra entre 50 y 54 años, y el menor entre 55 y 64 años. La experiencia en la práctica docente en educación superior va desde 0 años hasta más de 16 años, aunque la mayoría, el 24 \% (12), se encuentra en el rango entre 0 y 3 años, y la minoría, el 8 \% (4), entre 15 y 16 años; el promedio general de experiencia docente en educación superior es de 8,33 años.

En cuanto al nivel de educación de los docentes, se destaca que el $52 \%$ (26) son especialistas, el 32 \% (16) únicamente tienen formación de nivel profesional, el $10 \%$ (5) son magíster y el $2 \%$, es decir, un docente, ha terminado sus estudios doctorales. De otro lado, se observa que el $34 \%$ (17) de los docentes hace parte del programa de
Con el fin de cumplir el propósito de la investigación, se presenta el análisis estadístico de la información que se llevó a cabo: un análisis descriptivo de las variables que representan la información y un análisis de independencia Chi-cuadrado, que es el más adecuado por la naturaleza de los datos: se calcula el coeficiente de correlación de Pearson después de haber transformado las variables de cualitativas a cuantitativas $y$, luego, se definen las conclusiones a las que se llega.

Administración de Empresas (vale la pena aclarar que este es el programa con mayor número de estudiantes), el $28 \%$ (14) del programa de Administración de la Seguridad Social, el 20 \% (10) de Contaduría Pública y el 18 \% (9) del programa de Ingeniería de Sistemas (este es el programa con menor cantidad de estudiantes). En función de la modalidad en que dictan sus clases, se observa que el 52 \% (26) de los docentes trabajan en las modalidades presencial y distancia, el 38 \% (19) solo en la modalidad distancia y el $10 \%$ restante (5) en la modalidad presencial.

\section{Uso de herramientas web 2.0 en la educación superior}

La primera pregunta de investigación que se pensó para el desarrollo de artículo fue: ¿qué herramientas web 2.0 son utilizadas por los docentes como medio didáctico de apoyo a la formación del estudiante? Con base en esto, en primer lugar, a partir de las respuestas de los docentes, se determina que el 86 \% (43) manifestaron conocerlas, mientras que el $14 \%$ (7) no (ver figura 1). 
Figura 1. Respuestas a la pregunta “¿Conoce las herramientas web 2.0?"

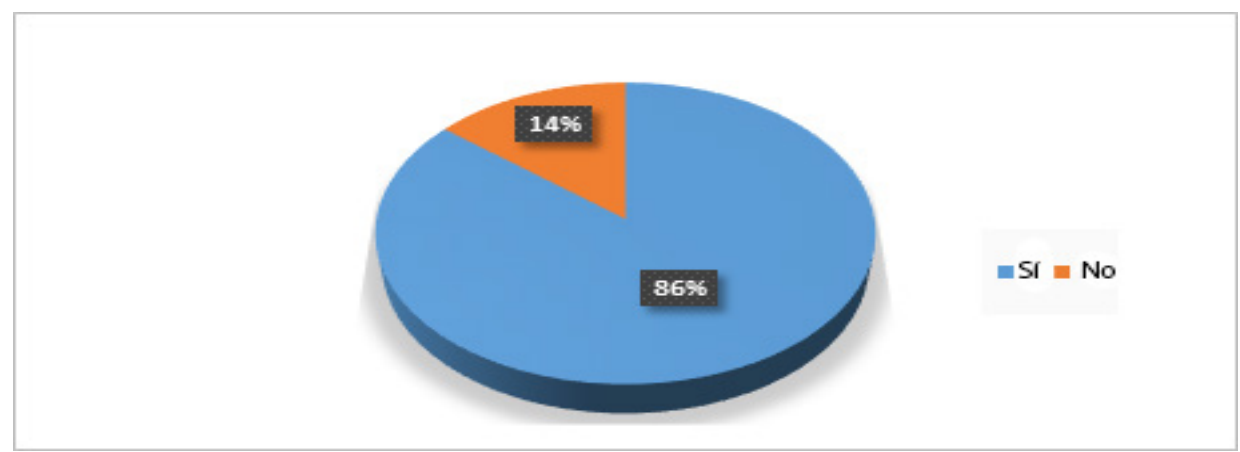

Fuente: elaboración propia.

En segundo lugar, considerando que los docentes pueden necesitar capacitaciones para aprender a utilizar de manera eficiente las herramientas web 2.0, se observa que el $52 \%$ (26) manifiesta no haber recibido ninguna capacitación, mientras que el 48 \% (24) sí lo ha hecho (ver figura 2).

Figura 2. Respuestas a la pregunta “¿Ha recibido capacitaciones sobre el uso de las herramientas web 2.0?"

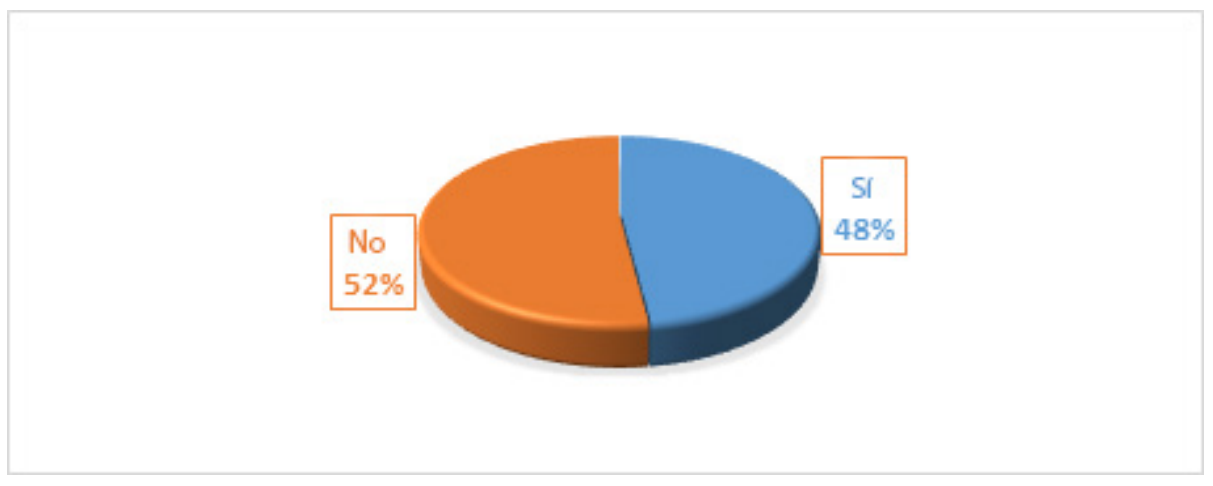

Fuente: elaboración propia.

En tercer lugar, de acuerdo con la pregunta de investigación sobre el uso de las herramientas web 2.0 en relación con el nivel de conocimiento, el $46 \%$ (23) de los docentes manifiesta tener un nivel básico, 38 \% (19) un nivel medio y solo el $16 \%$ (8) un nivel alto. Ahora bien, esto permite inferir que los docentes tienen disposición al uso de las herramientas web 2.0. 
Figura 3. Respuestas a la pregunta “¿Cómo definiría su nivel de conocimiento en el uso de las herramientas web 2.0?

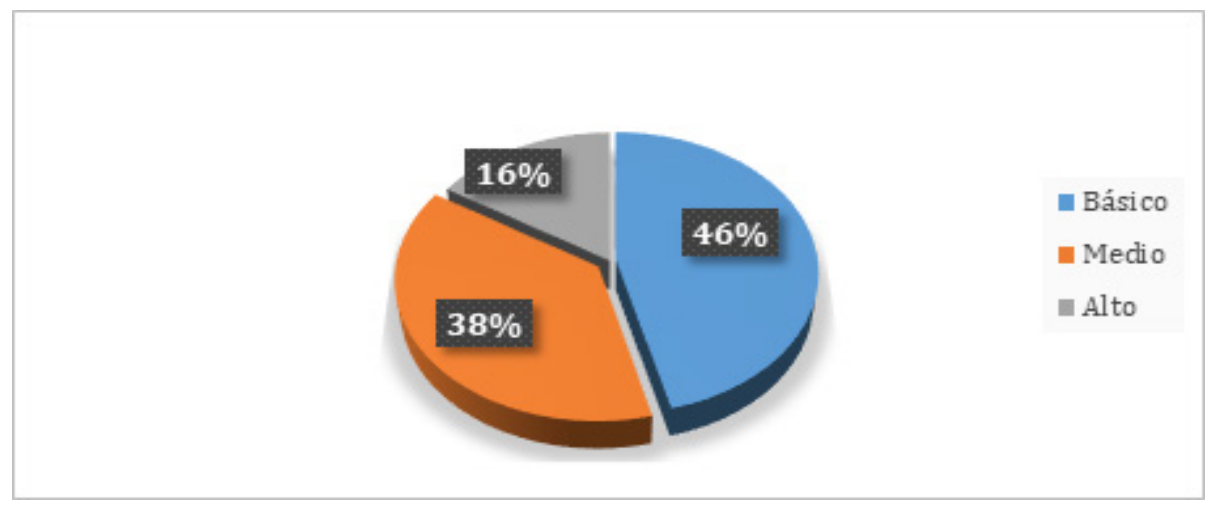

Fuente: elaboración propia.

En cuarto lugar, la frecuencia de uso de las herramientas web 2.0 por los docentes está determinada de la siguiente manera: el $56 \%$ (28) manifiesta que las utiliza casi siempre, el $28 \%$ (14) dice que siempre las usa, el $14 \%$ (7) afirma que muy pocas veces hace uso de ellas y solo un docente $(2 \%)$ indicó que nunca las utiliza. Esto nos indica que la mayoría de los docentes tiene la voluntad de utilizar estas herramientas.

Figura 4. Respuestas a la pregunta “¿Con qué frecuencia utiliza las herramientas web 2.0 en su labor como docente?

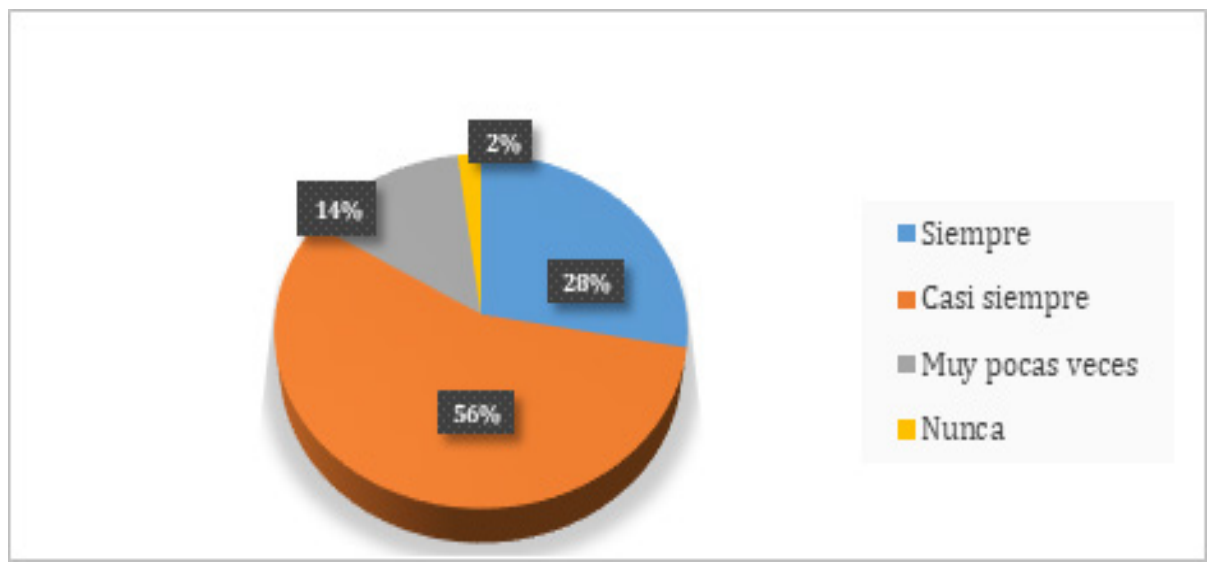

Fuente: elaboración propia.

En quinto lugar, en relación con las herramientas web 2.0 que se pueden utilizar en la práctica diaria, se evidencia que los blogs, las redes sociales y los servicios de video son las más conocidas por los docentes que participaron en la encuesta. Aquí, vale la pena recordar que estas fueron creadas con fines de comunicación masiva y no con un fin específico para la educación. Ahora bien, en los resultados que se incluyen en la figura 5, también se observa que las wikis y los mapas mentales son importantes para los docentes. 
Figura 5. Respuestas a la pregunta "¿De las siguientes herramientas web 2.0 para la práctica docente, cuáles conoce?

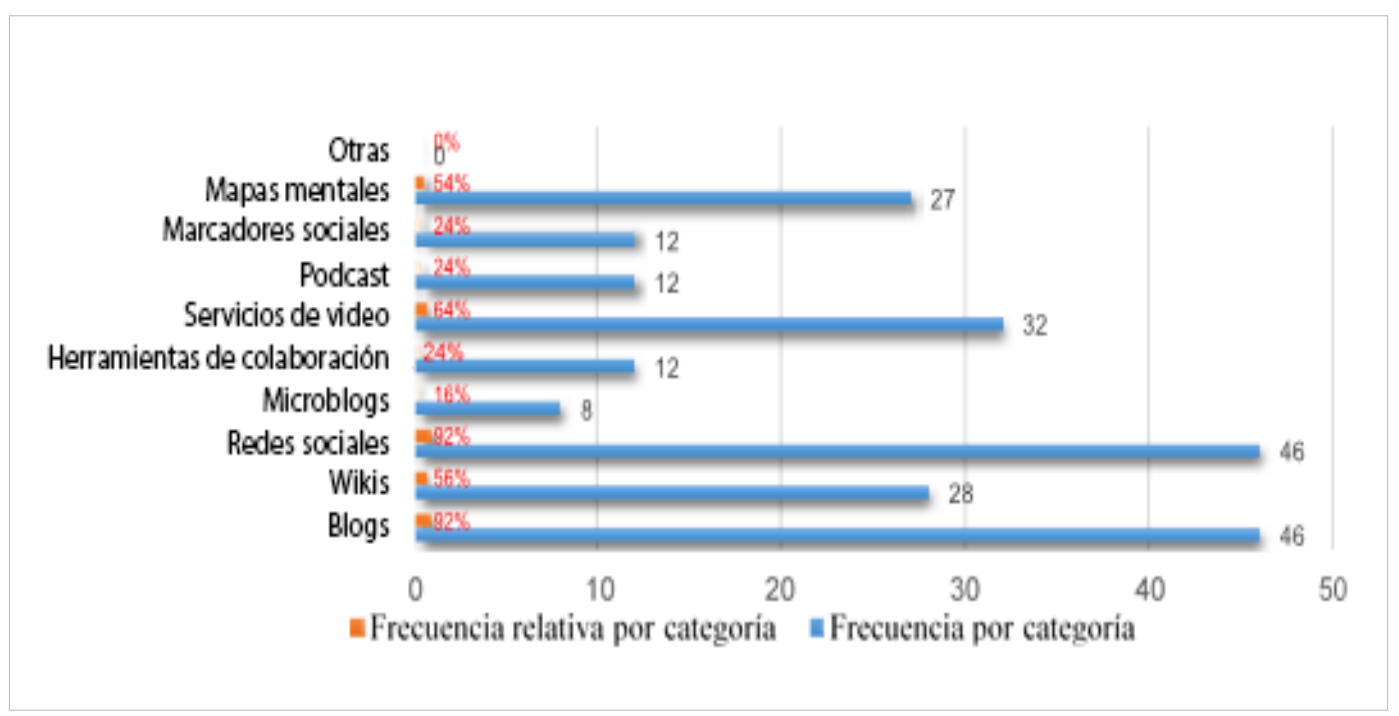

Fuente: elaboración propia.

En sexto lugar, se puede observar que las herramientas más usadas por los docentes son los blogs $(76 \%)$ y las redes sociales $(74 \%)$, mientras que las menos usadas son los marcadores sociales $(8 \%)$ y los microblogs (6\%) (ver figura 6). De otro lado, también es evidente que se mantiene una relación entre las herramientas que conoce el docente y las que usa en su labor. Igualmente, se aprecia que los docentes no utilizan las herramientas web 2.0 por la popularidad que estas tengan; lo hacen según las necesidades pedagógicas en su quehacer docente.

Figura 6. Respuestas a la pregunta “¿De las siguientes herramientas web 2.0 para la práctica docente, cuáles utiliza?

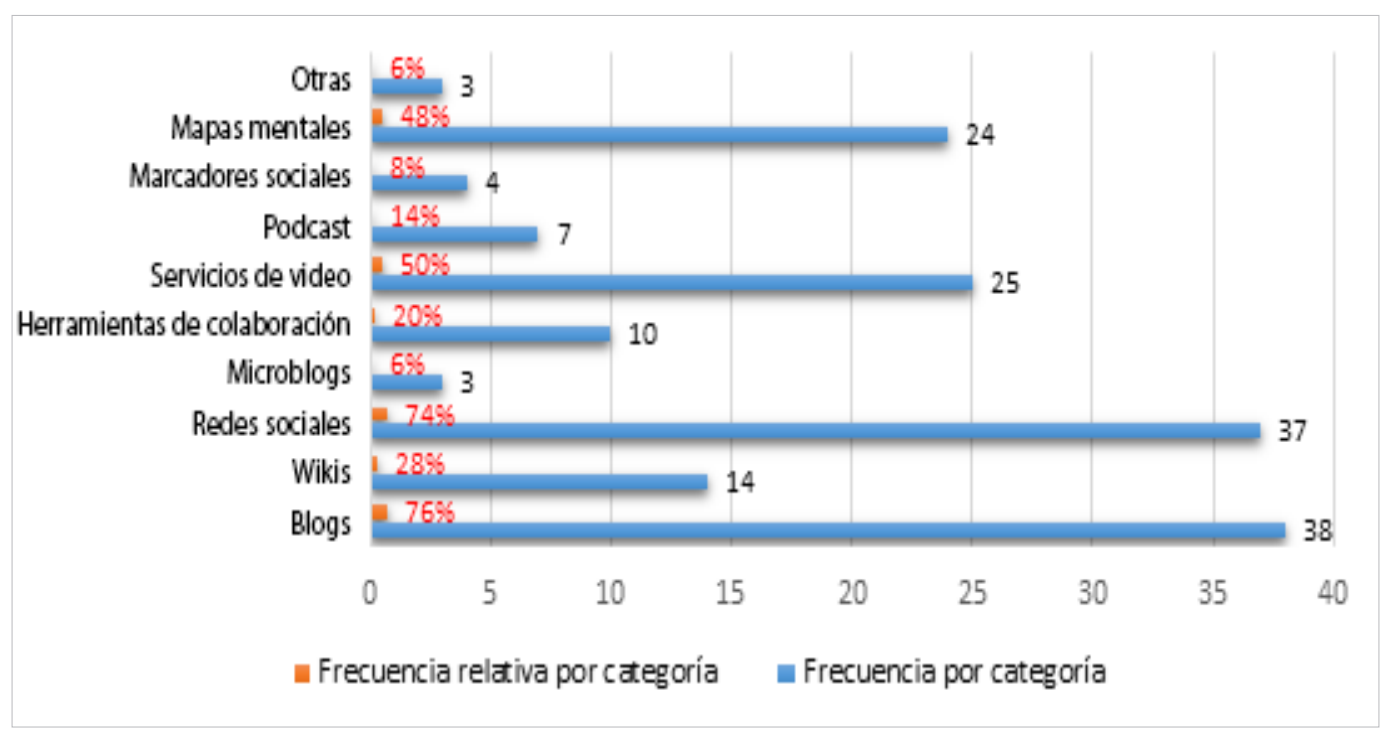

Fuente: elaboración propia. 
Finalmente, en séptimo lugar de este apartado, a partir de la encuesta practicada se puede afirmar que el 74 \% (37) prefiere utilizar, en el marco de su quehacer diario, las redes sociales, el $66 \%$ (33) los blogs, el 50 \% (25) los servicios de video y el 48 \% (24) los mapas mentales. Por otro lado, solamente el $8 \%$ (4) privilegia el uso de marcadores sociales y el $6 \%$ (3) prefiere trabajar con microblogs (ver figura 7).

Figura 7. Respuestas a la pregunta "¿De las siguientes herramientas web 2.0 para la práctica docente, cuáles prefiere utilizar?

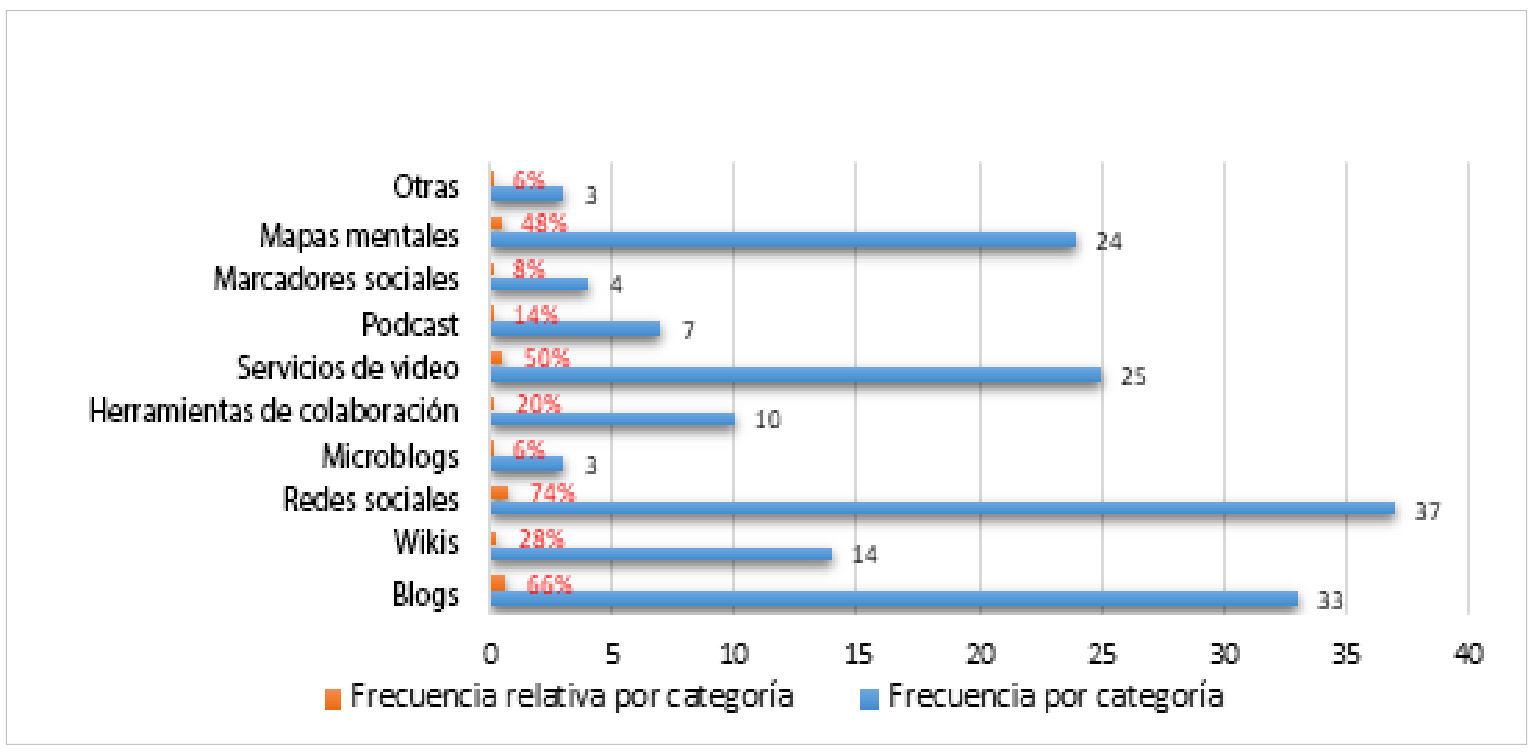

Fuente: elaboración propia.

\section{Uso constructivista de las herramientas web 2.0 en la educación superior}

La segunda pregunta de investigación en esta investigación fue: ¿cómo utiliza el docente las herramientas web 2.0 para la construcción de conocimiento?

A continuación, se sigue con el análisis descriptivo del uso de las herramientas web 2.0. En este apartado, el propósito es distinguir los usos que los docentes hacen de las herramientas web 2.0 para la construcción de conocimiento.

En este sentido, en la tabla 2 se observa la frecuencia de uso de blogs o microblogs. El 60 \%
(30) de los docentes afirmaron que los suelen utilizar como apoyo a los contenidos de clase, lo que evidencia el uso tradicional que le dan a estas herramientas web 2.0. Por su parte, el $14 \%$ (7) de los docentes indicaron que las utilizan para fomentar el debate y el 10 \% (5) para crear comunidades de aprendizaje. Es decir, dos de los fines indicados por los docentes son de carácter pedagógico, lo que demuestra el uso constructivista de las herramientas. Ahora bien,

también se observa que el 10 \% (5) de los docentes conocen los blogs o microblogs, pero no los usan, y que el 6 \% (3) no los conocen. 
Tabla 2. Frecuencias de usos de los blogs o microblogs en la práctica docente.

\begin{tabular}{|c|c|c|}
\hline Usos de los blogs o microblogs & $\begin{array}{c}\text { Frecuencia } \\
\text { absoluta }\end{array}$ & $\begin{array}{c}\text { Frecuencia } \\
\text { relativa }\end{array}$ \\
\hline Fomentar el debate & 7 & $14 \%$ \\
\hline Creación de comunidades de aprendizaje & 5 & $10 \%$ \\
\hline Apoyo a los contenidos de clases & 30 & $60 \%$ \\
\hline Sí los conozco, pero no los uso & 5 & $10 \%$ \\
\hline No los conozco & 3 & $6 \%$ \\
\hline Otro & 0 & $0 \%$ \\
\hline Total de observaciones & 50 & $100 \%$ \\
\hline
\end{tabular}

Fuente: elaboración propia.

En relación con el uso de las wikis por parte de los docentes, solo el $28 \%$ (14) participantes usan la herramienta como espacio de colaboración, mientras que el 8 \% (4) como espacio de creación de textos colaborativos. Estas son dos características constructivistas de la herramienta. Sin embargo, cabe destacar que el $26 \%$ (13) de los docentes conocen la herramienta, aunque no la usen, y que el $22 \%$ (11) manifiestan no conocerla (ver tabla 3). A partir de la encuesta desarrolla no se puede identificar por qué los docentes que conocen la existencia de las wikis no las utilizan.

Tabla 3. Frecuencias de usos de las wikis en la práctica docente.

\begin{tabular}{|c|c|c|}
\hline Usos de las wikis & $\begin{array}{l}\text { Frecuencia } \\
\text { absoluta }\end{array}$ & $\begin{array}{l}\text { Frecuencia } \\
\text { relativa }\end{array}$ \\
\hline Espacio de comunicación de la clase & 7 & $14 \%$ \\
\hline Espacio de colaboración & 14 & $28 \%$ \\
\hline Portafolio electrónico & 1 & $2 \%$ \\
\hline $\begin{array}{l}\text { Espacio de creación de textos } \\
\text { colaborativos }\end{array}$ & 4 & $8 \%$ \\
\hline Sí la conozco, pero no la uso & 13 & $26 \%$ \\
\hline No la conozco & 11 & $22 \%$ \\
\hline Otro & 0 & $0 \%$ \\
\hline Total de observaciones & 50 & $100 \%$ \\
\hline
\end{tabular}

Fuente: elaboración propia. 
De otro lado, en la tabla 4 se presentan las frecuencias del uso de las redes sociales. El 54 \% (27) de los docentes encuestados las utilizan, en su práctica docente, para compartir conocimientos entre alumnos; el $22 \%$ (11) para compartir documentos; el $10 \%$ (5) las conoce, pero no las usa; el $6 \%$ (3) las usa para fomentar la comunicación entre la familia y la escuela; el 4 \% (2) para compartir conocimientos entre docentes, el
$2 \%$ (1) para compartir fotografías, y el $2 \%$ (1) restante le da otro uso. Estos datos permiten determinar que esta herramienta web 2.0 se utiliza a partir de un objetivo comunicacional. En este sentido, se trata de una herramienta con un uso constructivista por parte de los docentes, ya que la utilizan desde los puntos de vista de interacción, conexión con el mundo real, reflexión con la experiencia y colaboración.

Tabla 4. Frecuencias de usos de las redes sociales en la práctica docente.

\begin{tabular}{|c|c|c|}
\hline Usos de las redes sociales & $\begin{array}{l}\text { Frecuencia } \\
\text { absoluta }\end{array}$ & $\begin{array}{l}\text { Frecuencia } \\
\text { relativa }\end{array}$ \\
\hline Compartir documentos & 11 & $22 \%$ \\
\hline Compartir fotografías & 1 & $2 \%$ \\
\hline $\begin{array}{l}\text { Fomentar la comunicación entre la familia } \\
\text { y la escuela }\end{array}$ & 3 & $6 \%$ \\
\hline Compartir conocimiento entre docentes & 2 & $4 \%$ \\
\hline Compartir conocimientos entre alumnos & 27 & $54 \%$ \\
\hline Sí las conozco, pero no las uso & 5 & $10 \%$ \\
\hline No las conozco & 0 & $0 \%$ \\
\hline $\begin{array}{l}\text { Otro (compartir conocimiento docente- } \\
\text { alumno) }\end{array}$ & 1 & $2 \%$ \\
\hline Total de observaciones & 50 & $100 \%$ \\
\hline
\end{tabular}

Fuente: elaboración propia.

En relación con los servicios de video, el 68 \% (34) de los docentes que participaron en el estudio manifestaron que los utilizaban. Del total encuestado, el 50 \% (25) los usa en tutorías a distancia (uso de tipo tradicional), el $12 \%$ (6) para reuniones de grupos de investigación y el $6 \%$ (3) para registrar bitácoras producto de debate o diálogos. Estas dos últimas finalidades corresponden a un uso constructivista. De igual modo, cabe destacar que el $22 \%$ (11) de los docentes encuestados conocen este tipo de herramienta, pero no usan ninguna, y que el $4 \%$ (2) dice no conocerlas, mientras que el $6 \%$ (3) corresponde a otros usos (ver tabla 5). 
Tabla 5. Frecuencias de usos de los servicios de video en la práctica docente.

\begin{tabular}{|c|c|c|}
\hline Usos de los servicios de videos & $\begin{array}{l}\text { Frecuencia } \\
\text { absoluta }\end{array}$ & $\begin{array}{l}\text { Frecuencia } \\
\text { relativa }\end{array}$ \\
\hline Tutorías a distancia & 25 & $50 \%$ \\
\hline Reuniones de grupos de investigación & 6 & $12 \%$ \\
\hline $\begin{array}{l}\text { Registrar bitácoras producto de un debate } \\
\text { o diálogo }\end{array}$ & 3 & $6 \%$ \\
\hline Sí los conozco, pero no los uso & 11 & $22 \%$ \\
\hline No los conozco & 2 & $4 \%$ \\
\hline Otro & 3 & $6 \%$ \\
\hline Total de observaciones & 50 & $100 \%$ \\
\hline
\end{tabular}

Fuente: elaboración propia.

DEn relación con el uso de pódcast, en la tabla 6 se muestran los resultados de la encuesta. El $38 \%$ (19) de los docentes participantes no conoce esta herramienta; el 32 \% (16) la conoce, pero no la usa; el $10 \%$ (5) usa pódcast para difundir contenidos de audio; el 10 \% (5) para distribuir contenidos de forma regular y periódica, y el $10 \%$
(5) para diversificar los recursos de la enseñanza (ver tabla 6). Estos tres último usos señalan su finalidad de interacción y conexión con el mundo real, lo cual nos indica que la tendencia de uso para la construcción de conocimiento es bastante baja.

Tabla 6. Frecuencias de usos de los pódcast en la práctica docente.

\begin{tabular}{|c|c|c|}
\hline Usos de los pódcast & $\begin{array}{c}\text { Frecuencia } \\
\text { absoluta }\end{array}$ & $\begin{array}{c}\text { Frecuencia } \\
\text { relativa }\end{array}$ \\
\hline Difundir contenidos de audio & 5 & $10 \%$ \\
\hline Distribuir contenidos de forma regular y periódica & 5 & $10 \%$ \\
\hline Diversificar los recursos de enseñanza & 5 & $10 \%$ \\
\hline Sí la conozco, pero no la uso & 16 & $32 \%$ \\
\hline No la conozco & 19 & $38 \%$ \\
\hline Otro & 0 & $0 \%$ \\
\hline Total de observaciones & 50 & $100 \%$ \\
\hline
\end{tabular}


Al determinar las frecuencias de usos de las herramientas web 2.0 en la práctica docente, llama la atención el caso de los marcadores sociales. El 40 \% (20) de los docentes encuestados manifiestan no conocer la herramienta y el $24 \%$ (12) la conoce, pero no la usa. De otro lado, el $20 \%$ (10) dice que la usa para registrar bibliografías o banco de información y, 10 \% (5) para trabajo cooperativo (esto concuerda con el uso efectivo de la herramienta) y el $6 \%$ (3) de los docentes afirman que la utilizan para la elaboración de portafolios (esta no es la finalidad de uso de la herramienta) (ver tabla 7). Dado lo anterior, se puede inferir que el uso de esta herramienta es relativamente bajo, motivo por el cual habría que investigar, por ejemplo, por qué tantos docentes la conocen, pero no la utilizan.

Tabla 7. Frecuencias de usos de los marcadores sociales en la práctica docente.

\begin{tabular}{ccc}
\hline Usos de los marcadores sociales & Frecuencia absoluta & Frecuencia relativa \\
\hline Elaboración de portafolios & 3 & $6 \%$ \\
\hline Trabajo cooperativo & 5 & $10 \%$ \\
\hline Registrar bibliografías o banco de información & $10 \%$ & $20 \%$ \\
\hline Si los conozco, pero no los uso & 12 & $24 \%$ \\
\hline No los conozco & 20 & $40 \%$ \\
\hline Otro & 0 & $0 \%$ \\
\hline Total de observaciones & 50 & $100 \%$
\end{tabular}

Fuente: elaboración propia.

Finalmente, en la tabla 8 se presentan los resultados relacionados con las frecuencias de usos de los mapas mentales. Según los datos recolectados, el 34 \% (17) de los docentes encuestados los usa para aprendizaje continuo; el $20 \%$ (10) para explorar conocimientos previos; el 16 \% (8) para reflexión en la experiencia; el 14 \% (7) los conoce, pero no lo usa; el $12 \%$ (6) no los conoce, y el $4 \%$ (2) les da otro uso.

Tabla 8. Frecuencias de usos de los mapas mentales en la práctica docente.

\begin{tabular}{|ccc}
\hline Usos de los mapas mentales & Frecuencia absoluta & Frecuencia relativa \\
Explorar conocimientos previos & 10 & $20 \%$ \\
\hline Reflexión en la experiencia & 8 & $16 \%$ \\
\hline Aprendizaje continuo & 17 & $34 \%$ \\
\hline Sí los conozco, pero no los uso & 7 & $14 \%$ \\
\hline No los conozco & 6 & $12 \%$ \\
\hline Otro & 2 & $4 \%$ \\
\hline Total de observaciones & 50 & $\mathbf{1 0 0} \%$ \\
\hline
\end{tabular}




\section{Análisis del uso de las herramientas web 2.0 para la construcción de conocimiento}

A partir de esta investigación, se puede afirmar que, en promedio, el 17,25 \% de los docentes que participaron en la encuesta manifiesta conocer las herramientas web 2.0 propuestas en este estudio para la construcción de conocimiento, pero también manifiestan no usarlas. En este sentido, valdría la pena indagar por las causas que llevan a que decidan no utilizarlas. De igual modo, también sería importante identificar los motivos por los cuales el 15,25 \% de estos docentes no las conocen, de forma tal que se puedan generar espacios adecuados para su promoción y capacitación.

Sumado a lo anterior, se observa que los docentes tienen mayor preferencia por el uso de redes sociales, blogs o microblogs, y en menor medida por las wikis, ya que las primeras les brindan espacios de interacción, conexión, reflexión y colaboración, características del constructivismo social. Por último, podría decirse que el docente, cuando decide emplear las herramientas web 2.0, lo hace con el propósito de construir conocimiento, aunque no tenga claro si la herramienta que escoge es la adecuada para tal fin. Los hallazgos aquí encontrados coinciden, en cierta medida, con la visión de la institución de educación superior en la que se hizo el estudio: en esta se propende por el uso de las herramientas web 2.0 por parte de los docentes. En este sentido, valdría la pena hacer un estudio enfocado exclusivamente en encontrar la mejor manera de enseñarle a los docentes cómo escoger la herramienta web 2.0 que deben utilizar.

\section{Necesidades de capacitación en herramientas web 2.0}

La tercera pregunta de investigación que se pensó para el desarrollo de artículo fue: ¿qué necesidades de capacitación tienen los docentes para un buen manejo de las herramientas web 2.0? Para contestar este interrogante, primero, se observa si los encuestados han recibido capacitación en el uso de estas herramientas y, luego, se analizan las tres preguntas que conforman este bloque. En cuanto a capacitaciones en el uso de las herramientas web 2.0 que han recibido los docentes, en la figura 8 se muestra que el $52 \%$ (26) de los docentes no la han recibido, mientras que el 48 \% (24) manifiesta haber recibido al menos una capacitación.

Figura 8. Respuestas a la pregunta “ ¿Ha recibido al menos una capacitación en el uso de las herramientas web 2.0 para la práctica docente?

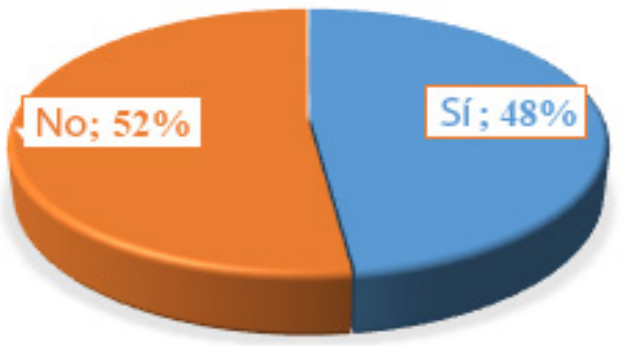

Fuente: elaboración propia. 
Por otra parte, de acuerdo con el nivel de satisfacción de los docentes en relación con las capacitaciones recibidas en herramientas web 2.0, el $32 \%$ (16) de los docentes manifiesta estar medianamente satisfecho; el $26 \%$ (13) dice no estar satisfecho; el $16 \%$ (8) afirman que están poco satisfechos, otro $16 \%$ (8) aseveran estar muy satisfechos, y el 10 \% (5) seleccionó la opción "otros" (ver tabla 9).

Tabla 9. Frecuencias de usos de satisfacción frente a las capacitaciones recibidas.

\begin{tabular}{lcc}
\hline $\begin{array}{c}\text { Nivel de satisfacción frente a } \\
\text { las capacitaciones }\end{array}$ & $\begin{array}{c}\text { Frecuencia } \\
\text { por categoría }\end{array}$ & $\begin{array}{c}\text { Frecuencia relativa } \\
\text { por categoría }\end{array}$ \\
\hline No satisfecho & 13 & $26 \%$ \\
\hline Poco satisfecho & 8 & $16 \%$ \\
\hline Medianamente satisfecho & 16 & $32 \%$ \\
\hline Muy satisfecho & 8 & $16 \%$ \\
\hline Otro & 5 & $10 \%$ \\
\hline
\end{tabular}

Fuente: elaboración propia.

\section{Conclusiones}

El uso de las TIC, y en especial el uso de las herramientas web 2.0, por parte de los docentes de las instituciones de educación superior se ha incrementado en la actualidad, gracias a que estas ofrecen recursos y herramientas que facilitan el acceso a la información e incentivan al alumnado a construir su aprendizaje, basados en la investigación y adecuación de los contenidos al entorno y a su propia realidad.

Este estudio está sujeto al uso de las herramientas web 2.0, al uso para la construcción de conocimiento y a las necesidades de capacitación que tienen los docentes. La información recolectada para este estudio está sustentada en un marco teórico estructurado y serio que otorga la validez y credibilidad que se requieren.
Dado a lo anterior se concluye que los factores de edad, nivel académico y conocimiento no son determinantes con el uso de las herramientas web 2.0. A partir de esto, se puede afirmar que cualquier docente tiene la posibilidad de dominar las herramientas web 2.0 que facilitan la práctica docente, pero, para lograr esto, es necesario tener la disposición para hacerlo. Lo anterior concuerda con la tesis de maestría titulada Uso de los servicios y herramientas de la web 2.0 por parte de los docentes de planta de la Universidad Tecnológica de Bolívar realizada por Acosta (2012). Por otra parte, queda demostrado que, a mayor nivel de conocimiento de la herramienta web 2.0, mayor será la frecuencia de uso. También es posible concluir que los docentes tienen mayor preferencia por el uso de redes sociales, blogs o microblogs, y en menor medida de las wikis. 
Esto se explica porque las primeras les brindan espacios de interacción, conexión, reflexión y colaboración, características del constructivismo social de Vigosky. Habría que hacer más estudios referentes al uso de las wikis para conocer la causa del poco uso, pues los docentes manifiestan conocerlas. Stefany Hernández (2008) confirma esta relación, al decir que, al darse un uso constructivista a estas nuevas tecnologías, se vinculan en la manera en que los alumnos aprenden mejor y son elementos importantes para la construcción de su conocimiento.

También puede decirse que el docente, cuando decide emplear las herramientas web 2.0, lo hace con el propósito de construir conocimiento, aunque no tenga claro si la herramienta que selecciona es la adecuada para tal fin. Como ya se dijo, valdría la pena hacer un estudio más profundo al respecto, ya que hay que tener en cuenta, por ejemplo, si el docente tiene formación pedagógica o no.

De otro lado, si se tiene en cuenta la gran variedad de herramientas web 2.0 disponibles en Internet para uso educativo, el nivel de conocimiento y aplicación de estas en las estrategias didácticas es bajo. Asimismo, es claro que, ante la ausencia de la aplicación por parte de los docentes en sus estrategias didácticas de las herramientas web 2.0, es muy probable que los estudiantes desconozcan su empleo y, por tanto, estén al margen de las muchas bondades que ellas ofrecen para su aprendizaje.

Manejar herramientas web 2.0 hace que el docente desarrolle una destreza fundamental, como lo es la adaptabilidad para seleccionar la herramienta más adecuada para cada propósito pedagógico. En este sentido, se debe conocer la amplia gama de aplicaciones disponibles y el o los usos pedagógicos en los cuales se especializa. El ideal es desarrollar en el docente una pedagogía crítica en la que el educador no solo utilice la herramienta, sino que también examine el objetivo y las ventajas de su aplicación en el aula.

Aunque la institución donde se hace el estudio tiene una política clara de formación en competencias TIC, de acuerdo con su modelo educativo, los docentes no reciben capacitaciones dirigidas hacia el uso de las herramientas web 2.0 que potencialicen el accionar docente en el uso de estas.

Esta investigación aporta a la creación de una base de conocimientos común sobre la manera en que se están utilizando herramientas de la web 2.0 en la docencia universitaria colombiana, específicamente en la ciudad de Montería, sus implicaciones para el proceso de enseñanza-aprendizaje y su influencia en la experiencia de los docentes.

También contribuye a que las directivas de la institución superior donde se hizo el estudio conozcan cómo utilizan las herramientas web 2-0 sus docentes y el uso que se les da para la construcción de conocimientos. En el ámbito nacional y mundial, el estudio contribuye al banco de conocimiento sobre la manera en que las herramientas web 2.0 se convierten en un apoyo para los docentes que utilizan una metodología constructivista.

Dentro de las limitaciones que tuvo el estudio, se encuentra un aspecto importante para tener presente, como lo es la muestra. Es importante tener en cuenta que, a partir de los docentes encuestas, no se puede generalizar al total de la población docente de la institución objeto de estudio, ya que el método de selección fue por conveniencia y no estratificado, y no se tiene una muestra representativa por cada programa. Además del método de selección de la muestra, vale la pena indicar que en la planeación de la investigación se aspiraba a contar con el apoyo de 93 docentes, pero 43 de ellos no diligenciaron la encuesta. En este punto, valdría la pena preguntarse por 
los motivos que no hicieron posible que el total de la muestra seleccionada respondiera la encuesta: ¿no tenían el tiempo suficiente para diligenciarla? ¿No consultaron su correo electrónico durante esos días? ¿No les interesaba el estudio?
Por último, es importante destacar que este estudio puede tener un mayor alcance en el marco de la región de Latinoamérica y del Caribe, con el objetivo de conocer, compartir y aprovechar las experiencias de colegas en contextos diferentes, lo cual podría contribuir a la mejora de la oferta académica en toda la región.

tecnológicas de sus docentes y prepararlos para la interacción pedagógica con ellas, sin dejar de lado la voluntad de gestionar recursos tendientes a la dotación de equipos y la infraestructura que hagan posible su empleo.

Finalmente, también se recomienda crear espacios de actualización docente interactiva, con una lista de opciones que permita escoger la posibilidad de integración de las herramientas web 2.0 al aula en relación con el contenido.

Lo importante no es utilizar la tecnología para que sustituya el aprendizaje y la enseñanza, sino que se convierta en un apoyo para ambas partes (Committee of Inquire into the Changing Learner Experience, 2009). mación en las instituciones de educación superior deben revisar, en gran medida, las fortalezas

\section{Referencias}

Acosta, J. (2012). Uso de los servicios y herramientas de la web 2.0 por parte de los docentes de planta de la Universidad Tecnológica de Bolívar (tesis de maestría). Universitat Oberta de Catalunya. Recuperado de https:/ / bit.ly/2Vj4CI9

Area, M. (2000). ¿Qué aporta internet al cambio pedagógico en la educación superior? En R. Pérez, M. Pascual, M. García, M. del Moral, M. Álvarez y B. Sierra (eds.), Redes, multimedia y diseños virtuales: Actas del III Congreso Internacional de Comunicación, Tecnología y Educación (pp. 128-135). Recuperado de https://bit.ly/3xnWxyW 
Boza, Á. y Conde, S. (2015). Web 2.0 en educación superior: formación, actitud, uso, impacto, dificultades y herramientas. Digital Education Review, 28, 45-58. Recuperado de https:/ / bit. ly/3fpnTyF

Cabero, J. (2001). Tecnología educativa. Diseño y utilización de medios en la enseñanza. Barcelona: Paidós.

Committee of Inquire into the Changing Learner Experience. (2009). Higher Education in a Web 2.0 World: Report of an independent Committee of Inquire into the impact on higher education of student's widespread use of Web 2.0 technologies. Recuperado de https:/ / bit.ly/2TQpQfX

Hernández, G. (2008). Los constructivismos y sus implicaciones para la educación. Perfiles Educativos, 30(122), 38-77. Recuperado de https:/ / bit.ly/2VefG9y

Hernández, R., Fernández, C. y Baptista, P. (2010). Metodología de la investigación. Ciudad de México: McGraw Hill Education.

Hernández, S. (2008). El modelo constructivista con las nuevas tecnologías: aplicado en el proceso de aprendizaje. Revista de Universidad y Sociedad del Conocimiento, 5(2), 26-35. Recuperado de https:// bit.ly/3ihdOWk

López, E. (2011). Metodología de la investigación: guía instruccional. Caracas: Universidad Nacional Abierta.

Rosario, J. (2006). TIC: su uso como herramienta para el fortalecimiento y el desarrollo de la educación virtual. DIM: Didáctica, Innovación y Multimedia, 8. Recuperado de https://bit. ly/3A1qExP

Zambrano, W. y Medina, V. (2010). Creación, implementación y validación de un modelo de aprendizaje virtual para la educación superior en tecnologías web 2.0. Signo y Pensamiento, 39(56), 288-303. Recuperado de https:// bit.ly/37cVxDl 